\title{
Hydrodynamic and label-free sorting of circulating tumor cells from whole blood
}

Cite as: Appl. Phys. Lett. 107, 203702 (2015); https://doi.org/10.1063/1.4935563

Submitted: 27 April 2015 • Accepted: 30 October 2015 • Published Online: 20 November 2015

Thomas M. Geislinger, Melanie E. M. Stamp, (D) Achim Wixforth, et al.

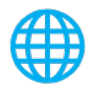

View Online
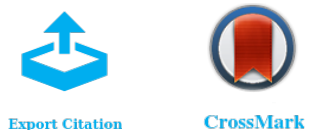

CrossMark

\section{ARTICLES YOU MAY BE INTERESTED IN}

Sorting of circulating tumor cells (MV3-melanoma) and red blood cells using non-inertial lift

Biomicrofluidics 7, 044120 (2013); https://doi.org/10.1063/1.4818907

Hydrodynamic mechanisms of cell and particle trapping in microfluidics

Biomicrofluidics 7, 021501 (2013); https://doi.org/10.1063/1.4799787

Experimental and numerical study of elasto-inertial focusing in straight channels

Biomicrofluidics 13, 034103 (2019); https://doi.org/10.1063/1.5093345

母QBLOX

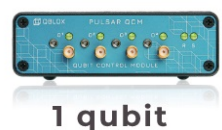

Shorten Setup Time Auto-Calibration More Qubits

Fully-integrated Quantum Control Stacks Ultrastable DC to $18.5 \mathrm{GHz}$ Synchronized $<<1$ ns Ultralow noise

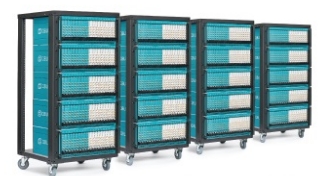

100s qubits

visit our website > 


\title{
Hydrodynamic and label-free sorting of circulating tumor cells from whole blood
}

\author{
Thomas M. Geislinger, ${ }^{1,2}$ Melanie E. M. Stamp, ${ }^{1,2}$ Achim Wixforth, ${ }^{1,2,3}$ \\ and Thomas Franke $1,2,3,4, a)$ \\ ${ }^{1}$ Experimental Physics I, Universität Augsburg, Universitätsstr. 1, 86159 Augsburg, Germany \\ ${ }^{2}$ Nanosystems Initiative Munich, Schellingstraße 4, 80799 München, Germany \\ ${ }^{3}$ Center for Nanoscience (CeNS), Ludwig-Maximilians-Universität München, Geschwister-Scholl Platz 1, \\ 80539 München, Germany \\ ${ }^{4}$ Chair of Biomedical Engineering, University of Glasgow, Oakfield Avenue, G12 8LT Glasgow, \\ United Kingdom
}

(Received 27 April 2015; accepted 30 October 2015; published online 20 November 2015)

\begin{abstract}
We demonstrate continuous, passive, and label-free sorting of different in vitro cancer cell lines (MV3, MCF7, and HEPG2) as model systems for circulating tumor cells (CTCs) from undiluted whole blood employing the non-inertial lift effect as driving force. This purely viscous, repulsive cell-wall interaction is sensitive to cell size and deformability differences and yields highly efficient cell separation and high enrichment factors. We show that the performance of the device is robust over a large range of blood cell concentrations and flow rates as well as for the different cell lines. The collected samples usually contain more than $90 \%$ of the initially injected CTCs and exhibit average enrichment factors of more than 20 for sorting from whole blood samples. (C) 2015 AIP Publishing LLC. [http://dx.doi.org/10.1063/1.4935563]
\end{abstract}

Metastasis is the major cause of cancer-related mortality but it is still unclear how it forms and progresses in detail. ${ }^{1}$ It is commonly assumed that the dissemination of the cancer from the primary tumor to distant organs is mediated by so-called circulating tumor cells (CTCs) ${ }^{2}$ in the bloodstream. They are of particular interest since they serve as a liquid, low-invasive biopsy from which prognostic information can be gathered, real-time therapy monitoring becomes possible, and in turn, therapeutic targets can be identified on the fly. ${ }^{3}$ Moreover, the examination of CTCs helps to improve our understanding of the metastatic process. ${ }^{3}$

Large numbers of CTCs in blood are known to correlate with an aggressive cancer progression by elevated metastasis and shorter times until relapse. This is especially true for, e.g., patients with progressive breast cancer. ${ }^{3-5}$ However, the term "large numbers" here denotes only about one to one hundred cells per milliliter blood! Thus, CTCs need to be enriched from peripheral blood samples to provide sufficiently many cells for any subsequent analysis. The only FDA (US Food and Drug Administration) approved method for this purpose is, so far, the CellSearch system (Veridex, USA). Here, magnetic particles, being functionalized by specifically designed antibodies, bind to the CTCs' membrane. A general disadvantage of such an immuno-labelling approach is that, usually, CTCs are not represented by a single, uniform cell type. Also, they are believed to undergo an epithelial-mesenchymal-transition (EMT) during which they actually lose their epithelial surface markers. Moreover, among them circulating cancer stem cells (CSCs) are also found which have no epithelial markers at all. ${ }^{1,4,6}$ Thus, it is very likely that there are many more CTCs in the blood stream than are detected by antibody-based methods.

\footnotetext{
a) Author to whom correspondence should be addressed. Electronic mail: thomas.franke@glasgow.ac.uk
}

To overcome this limitation of immuno-labelling techniques, intrinsic physical markers like cell size and deformability are exploited for label-free cell sorting. To enhance and optimize the sensitivity of sorting devices with respect to such parameters, miniaturization of any processes involved toward microfluidic devices have been proven to be extremely advantageous. ${ }^{7,8}$ Several approaches have been developed in the past, some of which are already summarized in various excellent review articles. ${ }^{9-16}$ In general, they can be classified into "active" and "passive" methods. While active methods rely on external (e.g., acoustic, 6,17,18 magnetic, ${ }^{19}$ and dielectrophoretic ${ }^{20,21}$ ) force fields for the separation of otherwise labelled cells, we here focus on passive, non-invasive, and label free methods that only exploit the hydrodynamic interaction between the cells, the channel structures, and the flow field. ${ }^{22-27}$

A very intuitive and well established passive approach for cell sorting according to size are micro-filter devices. ${ }^{22}$ An advanced realization of this principle is deterministic lateral displacement that uses arrays of obstacles to deflect cells into different outlets depending on cell size and deformability as intrinsic markers. ${ }^{23,28}$ Both methods filter or deflect cells using porous membranes or arrays of posts. The main challenge of such methods is clogging issues.

In sorting devices being based on purely hydrodynamic forces such as inertial forces or the non-inertial lift effect (NIL), we can waive such clogging-susceptible grids. ${ }^{29}$ All inertia-based devices are capable of high-throughput sample processing and perform very well for bead and solid microparticle separation, ${ }^{30}$ but, if deformability comes into play, their sorting performance decreases. ${ }^{31}$ This is a significant disadvantage for CTC sorting since CTCs are assumed to be the more deformable the higher their metastatic potential is. 1,4,14,32-34 Additionally, inertial approaches are mostly limited to low haematocrits, and the sorting performance 
decreases significantly with increasing cell concentration and decreasing diameter of the target cells. ${ }^{31}$ In spiral microchannels, CTCs can be sorted from sample solutions of higher haematocrits with high throughput and good sorting efficiencies. ${ }^{25,35}$ However, to be able to process whole blood samples in such devices, the cells need to be lysed. ${ }^{25}$ Since cell size is the crucial parametric marker for the inertial force-based separation, so far mostly the relatively large MCF7 cell line has been studied and the results deteriorate for smaller or more deformable cell lines. ${ }^{25,31,35}$

In contrast to inertial microfluidics, ${ }^{36}$ cells can also be sorted at very low Reynolds numbers employing the NIL. ${ }^{24,37}$ NIL is a hydrodynamic effect of purely viscous origin acting on soft, deformable objects under creeping (Stokes) flow conditions. ${ }^{38}$ The symmetry of a Stokes flow field can be broken by a deformable, non-spherical object, and the interaction of the perturbation with an adjacent channel wall then leads to a repulsive cell-wall-interaction. ${ }^{39-44} \mathrm{Olla}^{45}$ gave an approximate analytical solution for the NIL velocity of vesicles in a linear shear flow for large distances between cell and wall $(\mathrm{z} \gg \mathrm{R})$

$$
\frac{d z}{d t}=v_{l}(z)=\frac{\dot{\gamma} R^{3}}{z^{2}} U\left(\lambda, r_{1}, r_{2}\right) .
$$

Here, $R$ denotes the object radius, $z$ is the distance between the object's center of mass and the wall, and $U\left(\lambda, r_{1}, r_{2}\right)$ represents a dimensionless drift velocity which depends on the viscosity contrast $\lambda=\eta_{\text {in }} / \eta_{\text {ext }}$ between the internal viscosity of the vesicle (inner fluid + membrane) $\eta_{\text {in }}$ and the external fluid viscosity $\eta_{\text {ext }}$ as well as its shape. The latter is described by $r_{i}=a_{i} / a_{3}$ with the elliptical axes $a_{i}$. It has been shown that Olla's theoretical predictions are in very good agreement with experiments on vesicles, ${ }^{46}$ red blood cells (RBCs), and blood platelets. ${ }^{37}$ The differences in the lift velocity for cells of different sizes $^{24}$ and different deformability ${ }^{47}$ can be exploited with the method of non-inertial lift induced cell sorting (NILICS). ${ }^{24}$ This approach is more sensitive to deformability and operates at lower shear rates than inertial methods and has been shown to efficiently sort relatively small cancer cells (MV3 melanoma, diameter $\sim 14 \mu \mathrm{m}$ ) from RBC suspensions. $^{24}$

Here, we demonstrate that NILICS is even capable of sorting cancer cells from undiluted whole blood at stable and excellent sorting efficiencies. We investigate the influence of the flow rate ratio and use three in vitro cancer cell lines (MV3, MCF7, and HEPG2) to examine the sorting performance for target cells of different sizes and from diverse primary cancerous tissues. We find the sorting performance to be stable for different blood cell concentrations, flow rate ratios, and cell diameters. This robustness proves to be advantageous for real applications of cancer cell sorting and for the on-chip integration of the device.

For the RBC suspensions, we use freshly drawn blood from healthy, voluntary donors, and add ethylenediaminetetraacetic acid (EDTA) as anticoagulant. The blood sample is washed three times in isoosmotic phosphate buffered saline (PBS, $\mathrm{pH}=7.4$ ) to remove blood platelets, blood plasma, and white blood cells. The solution is then centrifuged at $\sim 70 \mathrm{~g}$ for $2 \mathrm{~min}$, and the recovered $\mathrm{RBC}$ pellet $(\mathrm{Hct} \approx 100 \%$ ) is finally diluted with PBS to the desired haematocrits (Hct $=20 \%, 30 \%$, and 40\%). The whole blood samples are only treated with the anticoagulant EDTA without any further preparation step.

Three aggressive, in vitro cancer cell lines from different original tissues are employed to test and confirm the sorting capability of our microfluidic device. We use MV3 melanoma cells with an average diameter of $(14 \pm 2) \mu \mathrm{m}$, the breast cancer line MCF7 (diameter: $(22 \pm 4) \mu \mathrm{m})$, and the hepatocellular carcinoma cell line HEPG-2 (diameter: $(13 \pm 2) \mu \mathrm{m})$, which we maintain according to standard cell culture protocols.

For the sample suspensions, we prepare a 6.5/3.5 mixture of PBS and OptiPrep (Axis-Shield) to match the fluid and cell densities and to avoid sedimentation. We added $2 \mathrm{mg} / \mathrm{ml}$ bovine serum albumin (BSA) and $5 \mathrm{mg} / \mathrm{ml}$ EDTA to prevent cell agglomeration and unspecific adhesion. After ultrasonic degassing, we suspend about one million cancer cells per milliliter together with RBCs from the pellet in ratios of $4 / 1,7 / 3$, and $3 / 2$ to ensure the final haematocrits of $20 \%, 30 \%$, and $40 \%$, respectively. For the whole blood samples, we also spike the untreated whole blood with about one million cancer cells per milliliter.

For the sheath flow, we prepare a solution of PBS and 5\% w/w dextrane (MW: 400-500 kDa, Sigma Aldrich Inc.) yielding a dynamic viscosity of $\eta_{\text {ext }}=7 \mathrm{mPas}$ at room temperature. To prevent pulsing effects in the microchannel, we adjust the concentration of Optiprep to the one in the sample solution that is: $1.2 \mathrm{ml}$ OptiPrep to $2.8 \mathrm{ml} \mathrm{PBS} /$ dextrane for the $\mathrm{Hct}=20 \%$ sample solution, $2.3 \mathrm{ml} \mathrm{PBS} /$ dextrane for $\mathrm{Hct}=30 \%, 1.8 \mathrm{ml} \mathrm{PBS} /$ dextrane for $\mathrm{Hct}=40 \%$, and $1.5 \mathrm{ml}$ for the whole blood sample, respectively. After another degassing step, we transfer the solution into Hamilton gastight syringes.

The experimental setup consists of a simple, singlelayer polydimethylsiloxane (PDMS) microchannel fabricated by standard soft lithography. ${ }^{48}$ It is mounted onto an inverted Zeiss Axiovert $200 \mathrm{~m}$ video microscope. A Photron Fastcam 1024 PCI records the images at $40 \times$ magnification at a speed of 125 frames per second to be able to determine the sorting efficiency, the enrichment factor, and the purity of the cancer cells.

The syringes are connected to the microchannel through polytetrafluoroethylene (PTFE) tubes. We drive the flows by two independent syringe pumps (PHD2000, Harvard Apparatus) at a constant sample flow rate of $\mathrm{Q}_{\text {sample }}=20 \mu \mathrm{l} / \mathrm{h}$ and variation of the sheath flow $\mathrm{Q}_{\text {sheath }}=400 \mu \mathrm{l} / \mathrm{h}, 600 \mu \mathrm{l} / \mathrm{h}$, and $800 \mu \mathrm{l} / \mathrm{h}$ to yield flow rate ratios of $\mathrm{Q}$-ratio $=\mathrm{Q}_{\text {sheath }} /$ $\mathrm{Q}_{\text {sample }}=20,30$, and 40, respectively. For $\mathrm{Q}_{\text {sample }}=20 \mu \mathrm{l} / \mathrm{h}$ and an assumed number of $\sim 6 \times 10^{9}$ cells per milliliter of whole blood, the throughput of the device lies in the range of $\sim 10^{8}$ cells per hour. Given our channel dimensions and the fluid viscosity ( $\eta_{\text {ext }}=7 \mathrm{mPas}$ ), we end up at low Reynolds numbers of $0.3<\operatorname{Re}<0.6$, and thus are safe to neglect any inertial effects in all presented experiments. ${ }^{36,49}$

Sample suspension and sheath flow solution are injected into the microchannel through two equally sized $(66 \mu \mathrm{m}$ $\times 63 \mu \mathrm{m})$ inlets. They converge perpendicularly to each other into the $20 \mathrm{~mm}$ long main channel of equal cross-section (see supplementary Figure S1 for a complete sketch ${ }^{50}$ ). $\mathrm{Q}_{\text {sample }}$ is 
focused to the lower wall by an appropriate $\mathrm{Q}_{\text {sheath }}$ and, for sufficiently high sheath flow rates, pinching occurs. This pinch effect forces the cells to flow very close to the wall such that their size determines the respective distance of their center of mass to the wall. ${ }^{51}$ As described earlier ${ }^{24}$ the pinch effect and the different sizes of RBCs and CTCs lead to a size-based pre-separation of the cell populations. This initially only marginal height difference is then, further downstream, drastically enhanced by the NIL effect. Being larger in general, ${ }^{52}$ CTCs from epithelial tumors exhibit a significantly higher lift velocity than the RBCs and thus, towards the end of the microchannel, align closer to the channel center. To technically facilitate the sorting step, we widen the microchannel in z-direction under an angle of $27^{\circ}$ up to a final height of $276 \mu \mathrm{m}$. This slanting height of the channels leads to a significant amplification of the height difference between the cells ${ }^{13}$ and enables sorting of the cancer cells into outlet 1 and of the RBCs into outlet 2 as depicted in Figure 1. The disjunction between outlets 1 and 2 is placed at a height of $84 \mu \mathrm{m}$, and the height offset of the cell stream in $\mathrm{z}$-direction is adjusted by the static pressure applied by the adjustable reservoirs connected to the outlets.

In previous experiments with an NILICS setup, ${ }^{24}$ we found high sorting efficiencies for MV3 cells spiked in low haematocrit $(\mathrm{Hct} \leq 9 \%)$ blood suspensions. While we found the sorting performance to be independent of the Qratio at very low haematocrits, it turned out to be Q-ratio dependent for $\mathrm{Hct}=9 \%$ due to the onset of cell-cellinteractions. ${ }^{24}$

We here extend these studies to higher blood cell concentrations (Hct $>9 \%$ ) and even up to whole blood. We examine the influence of the Q-ratio on the sorting performance of higher concentrated sample solutions, in which cellcell-interactions will continuously increase by injecting a mixture of MV3 cells spiked in a $\mathrm{Hct}=40 \%$ RBC-solution and investigating the cells at three different Q-ratios of 20 , 30 , and 40 .

The sorting performance of the device is parametrized using the sorting efficiency

$$
E=\frac{n_{c t c 1}}{n_{c t c}}
$$
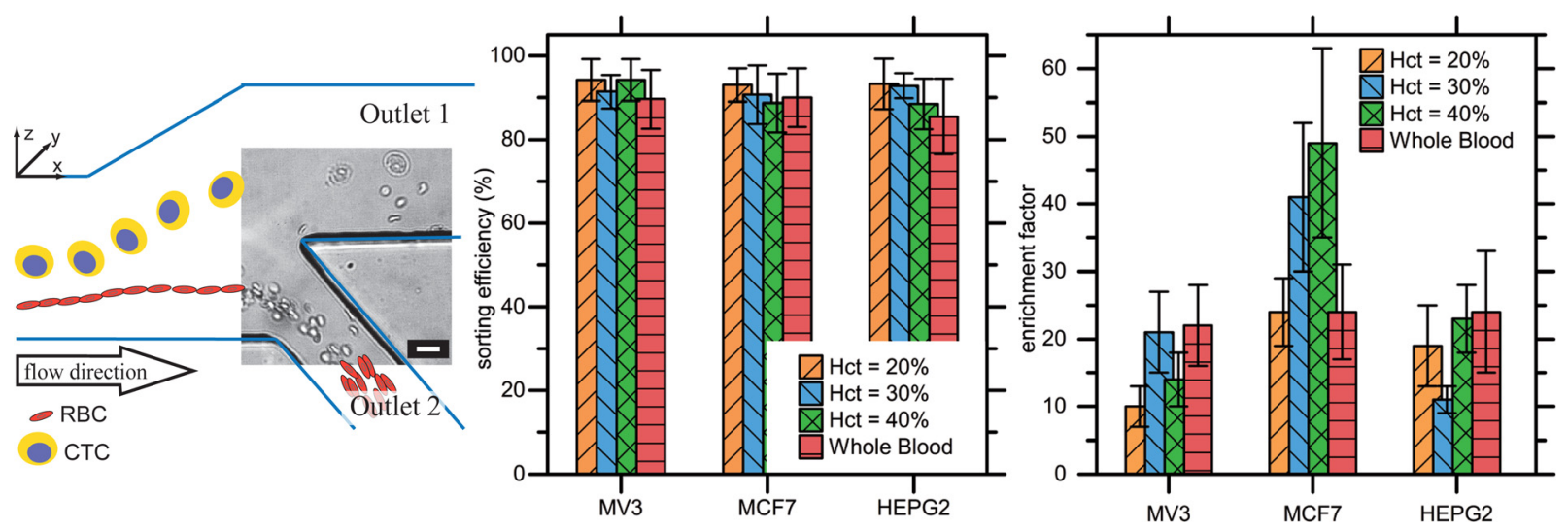

FIG. 1. Schematic drawing of the end of the microchannel with a micrograph of the sorting process (scale bar for the micrograph $=20 \mu \mathrm{m}$ ) and the sorting efficiencies together with enrichment factors for MV3, MCF7, and HEPG2 cells at a constant Q-ratio of $\mathrm{Q}_{\text {sheath }} / \mathrm{Q}_{\text {sample }}=600 / 20=30$ for different haematocrits $(\mathrm{Hct}=20 \%, 30 \%, 40 \%)$ and whole blood. The error bars represent the $95 \%$ confidence interval. A sketch of the complete microchannel is shown in the supplementary Figure S1 and the exact values for the sorting efficiencies and enrichment factors are summarized in supplementary Table $\mathrm{S} 1^{50}$ ). (Multimedia view) [URL: http://dx.doi.org/10.1063/1.4935563.1] the purity

$$
P=\frac{n_{c t c 1}}{\left(n_{c t c 1}+n_{r b c 1}\right)},
$$

and the enrichment factor

$$
A=\frac{n_{c t c 1} n_{r b c}}{n_{c t c} n_{r b c 1}}=E \times \frac{n_{r b c}}{n_{r b c 1}}
$$

as characteristic parameters. Here, $n_{c t c}$ and $n_{c t c l}$ denote the number of injected cancer cells and cancer cells in outlet 1 , $n_{r b c}$ the injected RBCs and $n_{r b c l}$ their number found in outlet 1 , respectively.

In all our experiments, we constantly find high sorting efficiencies of $\mathrm{E}_{\mathrm{Q} \text {-ratio }=20}=(96 \pm 5) \%, \mathrm{E}_{\mathrm{Q} \text {-ratio=30 }}=(94 \pm 5) \%$, and $\mathrm{E}_{\mathrm{Q} \text {-ratio=40 }}=(96 \pm 4) \%$ and no significant differences in purity or enrichment. ${ }^{50}$ The errors represent the $95 \%$ confidence interval. This is consistent with our previous measurements and demonstrates that a Q-ratio of 20 is well sufficient to achieve a proper pinch effect for Hct $=40 \%$. This finding is valid for lower haematocrits and is consistent with a hydrodynamic point of view: the volume of the channel is partitioned among $\mathrm{Q}_{\text {sheath }}$ and $\mathrm{Q}_{\text {sample }}$ according to the Qratio. Thus, the sample flow is confined to a height interval of $\Delta \mathrm{z} \leq 3.15 \mu \mathrm{m}$ for a Q-ratio of 20. This is sufficient to focus the RBCs below the center of mass of the CTCs and us to yield a pre-separation according to size. ${ }^{53}$ This prearation being produced by the pinch effect also helps to avoid unwanted collisions between the faster migrating CTCs and the RBCs during the separation process. ${ }^{24}$

For comparison, we also examine the sorting efficiency of MV3 cells at $\mathrm{Hct}=20 \%, 30 \%, 40 \%$, and whole blood at a constant $\mathrm{Q}_{\text {sheath }} / \mathrm{Q}_{\text {sample }}=30$ to evaluate possible influences of the increasing cell concentration on the sorting efficiency. We find constantly high sorting efficiencies for all sample suspensions as shown in Figure 1 (left). This demonstrates hat, for sufficiently high Q-ratios, the sorting performance is independent of the haematocrit of the sample suspension.

We now test the robustness of the sorting capability by using two additional cell lines differing in size (MCF7) and tissue type (MCF7 and HEPG2), while working at the same constant Q-ratio. While the MV3 melanoma cells and the 
HEPG2 hepatocellular carcinoma cells have similar sizes, the MCF7 breast cancer cells are larger. As illustrated in Figure 1, we find nearly constant sorting efficiencies for all cell types and sizes at all haematocrits and see no significant influence on the sorting efficiency. We thus can state that the sorting efficiency of our microfluidic device ranges up to an average of $\mathrm{E} \approx 90 \%$ for all cell types being nearly independent of the haematocrit from $\mathrm{Hct}=20 \%$ up to whole blood.

To characterize the sorting capability of the device in more detail, we now measure the purity $P$ and the enrichment factor $A$ for the collected samples. The measurements result in quite low purities $(0.1 \% \leq \mathrm{P} \leq 1 \%)$ but reasonable enrichment factors of $(10 \pm 3) \leq \mathrm{A} \leq(49 \pm 14)$ as illustrated in Figure 1 (right). Since the enrichment factor $A$ is determined from four single experimental parameters, the $95 \%$ confidence intervals of the values are quite large, and we cannot clearly resolve the dependence of the enrichment factor $A$ on the haematocrit. However, since the efficiency $E \approx$ const. $\approx 0.9$ for all experiments, as can be concluded from Figure 1 (left), the enrichment factor $A=E \times n_{r b c} / n_{r b c 1}$ only depends on the relative number of RBCs collected in outlet $1, n_{r b c 1}$, and the Hct of the injected suspension. In other words: A is determined by the RBCs that are dragged away into outlet 1 . A reasonable assumption would be to assume $n_{r b c 1}$ to be proportional to the number of the injected RBCs $n_{r b c}$, implying $A=$ const. if $E=$ const.. This is true for the MV3 and the HEPG2 cell lines, since the enrichment of both falls in the interval $20 \pm 5$, corresponding to about $5 \%$ of the RBCs that cross over to outlet 1 . However, the value for MCF7 cells $(A \approx 40)$ is significantly higher, meaning that only about $2.5 \%$ of the RBCs cross over to outlet 1 . MCF7 cell size is about 1.5 times the size of MV3 and HEPG2 cells and may have an effect on $A$ either via enhanced steric interaction of the larger cells with RBCs or via increased biochemical interaction due to the larger surface area. However, for whole blood $A=23 \pm 1$, for all cell types and cell sizes measured. Focusing of cells by the sheath flow may also have an additional effect since larger cells are focused to a lesser extent compared with the smaller RBCs. This could also explain why the enrichment of MCF7 cells at various Hct is increased with respect to MV3 and HEPG2 cells.

Although $\sim 96 \%$ of the RBCs are rejected in the whole blood measurements, we still find only low sample purities $P$ for all tested cell lines and parameters. This is due to the large absolute number of RBCs in comparison with the quite rare cancer cells. The purity can be written as a function of the enrichment factor $P=A \times r /(1+A \times r)$, with $r=\frac{n_{c c c}}{n_{r b c}}$ and for small values of $\mathrm{r}$ as used in the experiments $\left(r \leq 10^{-3}\right)$ this relation simplifies to $P=A \times r$, which explains the small values for $\mathrm{P}$ in our measurements. Although for a single sorting run this seems to be unfavorable, it can be significantly improved by cascading. This can be achieved either via reinjection of the sample or by several sequential sorting channels employing standard microfluidic techniques. The purity in each sort increases by a factor $A$, since $r_{1}=P=A \times r$, with $r_{1}=\frac{n_{c c c 1}}{n_{r b c l}}$, this quickly approaches large purities of $P \approx 1$. Together with the high sorting efficiency meaning nearly no loss of cells per sorting run, this is a promising approach to further improve the sorting performance of the device.
The sorting efficiency is stable for a wide range of flow rates and yields comparable results for various cancer cell lines of different cancer cell sizes as small as $R \approx 13 \mu \mathrm{m}$. We thus expect an even better sorting performance for more deformable CTCs from patient samples. ${ }^{14}$ This is in contrast to the common assumption that higher deformability disturbs the sorting process. ${ }^{31,35}$ Compared with other methods, ${ }^{25,31,35,54}$ it is safe to state that our NILICS provides a reasonable trade between high sorting efficiencies and correspondingly good enrichment factors, especially for small and deformable CTCs.

In conclusion, we have demonstrated that the NILICS approach is excellently capable of sorting various cancer cells out of highly concentrated RBC suspensions and even undiluted whole blood samples. The examination of real patient samples and a further improvement of the sorting performance will be our next step to investigate the practical applicability of NILICS for on-chip CTC sorting from whole blood suspensions.

The authors thank Professor Stefan Schneider, Universitätsklinikum Mannheim, Ruprecht-Karls-Universität Heidelberg, for providing the MV3 and Professor Ernst Wagner, Ludwig-Maximilians-Universität, Munich, for providing the MCF7 and the HEPG2 cell lines via the Center for Nanoscience (CeNS). T.G. would like to thank Christoph Westerhausen for the help with data processing. T.F. thanks Erich Sackmann for numerous discussions. This work has been funded in part by the Deutsche Forschungsgemeinschaft under the framework of the Cluster of Excellence "Nanosystems Initiative Munich" and the project "Dynamics of collective phenomena in red blood cells" FR 2473 4-2.

${ }^{1}$ V. Plaks, C. D. Koopman, and Z. Werb, Science 341, 1186 (2013).

${ }^{2}$ Y. Shiozawa, A. M. Havens, K. J. Pienta, and R. S. Taichman, Leukemia 22, 941 (2008).

${ }^{3}$ C. Alix-Panabières and K. Pantel, Clin. Chem. 59, 110 (2013).

${ }^{4}$ C. L. Chaffer and R. A. Weinberg, Science 331, 1559 (2011).

${ }^{5}$ I. Baccelli, A. Schneeweiss, S. Riethdorf, A. Stenzinger, A. Schillert, V. Vogel, C. Klein, M. Saini, T. Bäuerle, M. Wallwiener, T. Holland-Letz, T. Höfner, M. Sprick, M. Scharpff, F. Marmé, H.-P. Sinn, K. Pantel, W. Weichert, and A. Trumpp, Nat. Biotechnol. 31, 539 (2013).

${ }^{6}$ P. Augustsson, C. Magnusson, M. Nordin, H. Lilja, and T. Laurell, Anal. Chem. 84, 7954 (2012).

${ }^{7}$ P. Li, Z. S. Stratton, M. Dao, J. Ritz, and T. J. Huang, Lab Chip 13, 602 (2013).

${ }^{8}$ T. A. Franke and A. Wixforth, Chemphyschem 9, 2140 (2008).

${ }^{9}$ L. Yu, S. R. Ng, Y. Xu, H. Dong, Y. J. Wang, and C. M. Li, Lab Chip 13, 3163 (2013).

${ }^{10}$ A. A. S. Bhagat, H. Bow, H. W. Hou, S. J. Tan, J. Han, and C. T. Lim, Med. Biol. Eng. Comput. 48, 999 (2010).

${ }^{11}$ I. Cima, C. Wen Yee, F. S. Iliescu, W. Min Phyo, K. Hon Lim, C. Iliescu, and M. Han Tan, Biomicrofluidics 7, 11810 (2013).

${ }^{12}$ K.-A. Hyun and H.-I. Jung, Lab Chip 14, 45 (2014).

${ }^{13}$ W. C. I. Shields, D. Reyes, and G. P. López, Lab Chip (2015).

${ }^{14}$ C. Jin, S. M. McFaul, S. P. Duffy, X. Deng, P. Tavassoli, P. C. Black, and H. Ma, Lab Chip 14, 32 (2014).

${ }^{15}$ A. Karimi, S. Yazdi, and A. M. Ardekani, Biomicrofluidics 7, 021501 (2013).

${ }^{16}$ M. Kersaudy-Kerhoas and E. Sollier, Lab Chip 13, 3323 (2013).

${ }^{17}$ T. Franke, S. Braunmüller, L. Schmid, A. Wixforth, and D. A. Weitz, Lab Chip 10, 789 (2010).

${ }^{18}$ V. Skowronek, R. W. Rambach, L. Schmid, K. Haase, and T. Franke, Anal. Chem. 85, 9955 (2013).

${ }^{19}$ N. Pamme and C. Wilhelm, Lab Chip 6, 974 (2006).

${ }^{20}$ X. Wang, J. Yang, Y. Huang, J. Vykoukal, F. F. Becker, and P. R. C. Gascoyne, Anal. Chem. 72, 832 (2000). 
${ }^{21}$ S. Shim, K. Stemke-Hale, A. M. Tsimberidou, J. Noshari, T. E. Anderson, and P. R. C. Gascoyne, Biomicrofluidics 7, 11807 (2013).

${ }^{22}$ P. Preira, V. Grandné, J.-M. Forel, S. Gabriele, M. Camara, and O. Theodoly, Lab Chip 13, 161 (2013).

${ }^{23}$ J. A. Davis, D. W. Inglis, K. J. Morton, D. A. Lawrence, L. R. Huang, S. Y. Chou, J. C. Sturm, and R. H. Austin, Proc. Natl. Acad. Sci. U.S.A. 103, 14779 (2006).

${ }^{24}$ T. M. Geislinger and T. Franke, Biomicrofluidics 7, 44120 (2013).

${ }^{25}$ M. E. Warkiani, G. Guan, K. B. Luan, W. C. Lee, A. A. S. Bhagat, P. K. Chaudhuri, D. S.-W. Tan, W. T. Lim, S. C. Lee, P. C. Y. Chen, C. T. Lim, and J. Han, Lab Chip 14, 128 (2014).

${ }^{26}$ D. Di Carlo, D. Irimia, R. G. Tompkins, and M. Toner, Proc. Natl. Acad. Sci. U.S.A. 104, 18892 (2007).

${ }^{27}$ M. Yamada, M. Nakashima, and M. Seki, Anal. Chem. 76, 5465 (2004).

${ }^{28}$ Z. Liu, F. Huang, J. Du, W. Shu, H. Feng, X. Xu, and Y. Chen, Biomicrofluidics 7, 011801 (2013).

${ }^{29}$ T. M. Geislinger and T. Franke, Adv. Colloid Interface Sci. 208, 161 (2014).

${ }^{30}$ J. Zhou, P. V. Giridhar, S. Kasper, and I. Papautsky, Lab Chip 13, 1919 (2013).

${ }^{31}$ E. Sollier, D. E. Go, J. Che, D. R. Gossett, S. O’Byrne, W. M. Weaver, N. Kummer, M. Rettig, J. Goldman, N. Nickols, S. McCloskey, R. P. Kulkarni, and D. Di Carlo, Lab Chip 14, 63 (2014).

${ }^{32}$ J. Guck, S. Schinkinger, B. Lincoln, F. Wottawah, S. Ebert, M. Romeyke, D. Lenz, H. M. Erickson, R. Ananthakrishnan, D. Mitchell, J. Käs, S. Ulvick, and C. Bilby, Biophys. J. 88, 3689 (2005).

${ }^{33}$ V. Swaminathan, K. Mythreye, E. T. O'Brien, A. Berchuck, G. C. Blobe, and R. Superfine, Cancer Res. 71, 5075 (2011).

${ }^{34}$ S. Suresh, Acta Mater. 55, 3989 (2007).

${ }^{35}$ H. W. Hou, M. E. Warkiani, B. L. Khoo, Z. R. Li, R. A. Soo, D. S.-W. Tan, W.-T. Lim, J. Han, A. A. S. Bhagat, and C. T. Lim, Sci. Rep. 3, 1259 (2013).

${ }^{36}$ D. Di Carlo, Lab Chip 9, 3038 (2009).
${ }^{37}$ T. M. Geislinger, B. Eggart, S. Braunmüller, L. Schmid, and T. Franke, Appl. Phys. Lett. 100, 183701 (2012).

${ }^{38}$ H. L. Goldsmith and S. G. Mason, Nature 190, 1095 (1961).

${ }^{39}$ I. Cantat and C. Misbah, Phys. Rev. Lett. 83, 880 (1999).

${ }^{40}$ U. Seifert, Phys. Rev. Lett. 83, 876 (1999).

${ }^{41}$ M. Abkarian, C. Lartigue, and A. Viallat, Phys. Rev. Lett. 88, 068103 (2002).

${ }^{42}$ S. Meßlinger, B. Schmidt, H. Noguchi, and G. Gompper, Phys. Rev. E 80, 011901 (2009).

${ }^{43}$ C. A. Stan, A. K. Ellerbee, L. Guglielmini, H. A. Stone, and G. M. Whitesides, Lab Chip 13, 365 (2013).

${ }^{44}$ R. Zhou, J. Gordon, A. F. Palmer, and H.-C. Chang, Biotechnol. Bioeng. 93, 201 (2006).

${ }^{45}$ P. Olla, J. Phys. II France 7, 1533 (1997).

${ }^{46}$ N. Callens, C. Minetti, G. Coupier, M.-A. Mader, F. Dubois, C. Misbah, and T. Podgorski, Europhys. Lett. 83, 24002 (2008).

${ }^{47}$ T. M. Geislinger, S. Chan, K. Moll, A. Wixforth, M. Wahlgren, and T. Franke, Malar. J. 13, 375 (2014).

${ }^{48}$ Y. Xia and G. M. Whitesides, Annu. Rev. Mater. Sci. 28, 153 (1998).

${ }^{49}$ H. Amini, E. Sollier, W. M. Weaver, and D. Di Carlo, Proc. Natl. Acad. Sci. U.S.A. 109, 11593 (2012).

${ }^{50}$ See supplementary material at http://dx.doi.org/10.1063/1.4935563 for a complete sketch of the setup and the numerical values of sorting efficiency and enrichment factor.

${ }^{51}$ C. Cupelli, T. Borchardt, T. Steiner, N. Paust, R. Zengerle, and M. Santer, Microfluid. Nanofluid. 14, 551 (2012).

${ }^{52}$ G. Vona, A. Sabile, M. Louha, V. Sitruk, S. Romana, K. Schütze, F. Capron, D. Franco, M. Pazzagli, M. Vekemans, B. Lacour, C. Bréchot, and P. Paterlini-Bréchot, Am. J. Pathol. 156, 57 (2000).

${ }^{53}$ A. Srivastav, T. Podgorski, and G. Coupier, Microfluid. Nanofluid. 13, 697 (2012).

${ }^{54}$ M. G. Lee, J. H. Shin, C. Y. Bae, S. Choi, and J. Park, Anal. Chem. 85, 6213 (2013). 